\title{
ESTUDIO DE PIGMENTOS EN ALFARERÍA ESTILO NEGRO SOBRE ROJO DE QUEBRADA DE HUMAHUACA, JUJUY, ARGENTINA
}

\section{STUDY OF PIGMENTS USED IN BLACK-ON-RED CERAMICS FROM QUEBRADA DE HUMAHUACA, JUJUY, ARGENTINA}

\author{
Verónica J. Acevedo*, Mariel A. LÓpez** \\ ELEONORA FreIRE* ** EMILIA B. HALAC $* * * \star$ \\ GRISELDA POLLA **** '\& María REINOSO $* * * * *^{\prime} *$
}

En las piezas cerámicas Negro sobre Rojo de la Quebrada de Humahuaca pueden observarse atributos tecnológicos que aportan información sobre su variabilidad local y cronológica. En este trabajo se realiza el análisis de pigmentos sobre muestras cerámicas procedentes de dicha región, mediante diversas técnicas arqueométricas. Algunas de uso ya común, como la microscopia óptica y la difracción de rayos X, y otras de más reciente aplicación, como la espectroscopia Raman y la microscopia electrónica de barrido con espectroscopia de energía dispersiva. La aplicación combinada de diversas técnicas y la discusión del caso con los distintos especialistas permitió establecer interpretaciones significativas a partir de los resultados obtenidos.

Palabras clave: cerámica Estilo Negro sobre Rojo, Quebrada de Humahuaca, DRX, espectroscopia Raman, SEM-EDS

Technological attributes that provide information about local and chronological variability can be observed in ceramic pieces Black-on-Red style from the Quebrada de Humabuaca. In this paper we offer an analysis of pigments from ceramic samples from that region that was carried out using a variety of arquaeometric techniques, including traditional ones such as optical microscopy and X-ray diffraction, as well as more recently developed ones such as Raman spectroscopy and SEM-EDS. The combined application of several archaeometric techniques and the case discussion with several experts yielded significant interpretations of the results obtained.

Keywords: Black-on-Red ceramic style, Quebrada de Humabuaca, XRD, Raman Spectroscopy, SEM-EDS

\section{INTRODUCCIÓN}

Desde principios del siglo xx, el análisis del material cerámico en el Noroeste Argentino estuvo vinculado a aspectos tipológicos y descriptivos relacionados con la forma y la decoración de piezas enteras, en general, pertenecientes a contextos funerarios. A partir de esto, se establecieron clasificaciones que fueron utilizadas como indicativas de grupos culturales adscribibles a ciertos períodos de tiempo. En la actualidad esta perspectiva ha sido revisada y criticada por varios investigadores en vista de nuevas líneas teóricas de análisis, con las cuales se desarrollan estudios sobre materiales cerámicos arqueológicos (Cremonte 1985, 1991, 2006; Rivolta 1997; López 2004; Cremonte \& Bugliani 2010; Acevedo 2011, entre otros).

Desde hace algo más de dos décadas han comenzado a aplicarse técnicas fisicoquímicas en el estudio de material cerámico, lo que ha contribuido considerablemente a resolver problemas arqueológicos referidos al origen, la producción, el uso y la depositación de piezas cerámicas (Cremonte 1985, 1991; López 2004).

* Verónica J. Acevedo, Instituto de Arqueología, Facultad de Filosofía y Letras, Universidad de Buenos Aires, 25 de Mayo 217 , 3 er piso of. 3 , (1002) Capital Federal, Argentina, email: veronicaacevedo@speedy.com.ar

* Mariel A. López, Instituto de Arqueología, Facultad de Filosofía y Letras, Universidad de Buenos Aires. Consejo Nacional de Investigaciones Científicas y Técnicas, Argentina, email: marielarqueologia@yahoo.com.ar

** Eleonora Freire, Gerencia de Investigación y Aplicaciones, Comisión Nacional de Energía Atómica. Universidad Nacional de San Martín. Consejo Nacional de Investigaciones Científicas y Técnicas, Argentina, email: freire@tandar.cnea.gov.ar

***: Emilia B. Halac, Gerencia de Investigación y Aplicaciones, Comisión Nacional de Energía Atómica. Universidad Nacional de San Martín, Argentina, email: halac@cnea.gov.ar

**:*: Griselda Polla, Gerencia de Investigación y Aplicaciones, Comisión Nacional de Energía Atómica, Argentina, email: grispoll@cnea.gov.ar

**** María Reinoso, Gerencia de Investigación y Aplicaciones, Comisión Nacional de Energía Atómica. Universidad Nacional de San Martín. Consejo Nacional de Investigaciones Científicas y Técnicas, Argentina, email: reinoso@cnea.gov.ar 
La tecnología cerámica, como estudio de la alfarería en términos de sus materiales y métodos de manufactura, es actualmente un campo especializado de la investigación arqueológica en el Noroeste Argentino (Cremonte 1985, 1991, 2006; López 2004; De la Fuente 2005; De la Fuente et al. 2007; Cremonte \& Bugliani 2010; Acevedo 2011, entre otros). La perspectiva de análisis de la tecnología cerámica desde la ciencia de los materiales implica un conjunto racionalmente ordenado de conocimientos científicos, conocimientos empíricos, técnicas y especificaciones de los medios de producción que permiten su fabricación. En este sentido, se estudian sus características, estructuras, propiedades, procesos de fabricación y comportamiento en todas las situaciones posibles (Mari 1998). Es decir, los procedimientos analíticos que se utilizan en este tipo de investigación refieren a estudios mineralógicos y fisicoquímicos, trabajos de experimentación y empleo de datos etnográficos. Los mismos pueden desarrollarse en forma conjunta o en razón de las disponibilidades y del tipo de información que se desea obtener para resolver determinados problemas arqueológicos (Cremonte 1985, 1991; López 2004; Cremonte \& Bugliani 2010; Acevedo 2011).

En este trabajo hacemos referencia al Estilo Negro sobre Rojo, enfocándonos en los aspectos tecnológicos de la cerámica analizada y tomando como referencia las líneas teóricas sobre tecnología cerámica expresadas anteriormente.

Las vasijas pintadas con motivos en negro sobre rojo son consideradas como el estilo característico de las sociedades de la Quebrada de Humahuaca. Este estilo se consolida durante la segunda fase del Período de Desarrollos Regionales (ca. 900-1430 DC) y perdura hasta la época poscontacto con el europeo (Nielsen 1997, 2001; Cremonte 2006). Según Nielsen (2010), se estima que las piezas diseñadas anteriores al 1200 DC, Período Desarrollos Regionales I (ca. 900-1250 DC), han sido regularmente encontradas en un área que excede al valle de Humahuaca, abarcando desde San Salvador hasta la Puna en la frontera de Argentina y Bolivia. En cambio, las posteriores a esta fecha se corresponderían con diseños propios de la Quebrada de Humahuaca.

Sin pretender otorgar a esos objetos el lugar de insignias identitarias, pareciera que en la sencilla experiencia de comer y beber de estas vasijas en el siglo XIII hubo un cambio en la concepción que las personas tuvieron de sí mismas, en la que establecen fronteras entre el parecerse y el diferenciarse (Nielsen 2010).

Hay que considerar que, si bien, el estilo es considerado como representativo de las sociedades que habitaron la Quebrada de Humahuaca en el Período de Desarrollos Regionales II (ca. 1250-1430 DC), hay variantes temporales y locales en lugares que, aunque cercanos, pudieron tener historias diversas (Cremonte 2006; Nielsen 2010). Por ello se considera que algunas de sus características podrían estar reflejando diferencias entre las entidades sociales de una región (Cremonte 2006). Si bien visualmente este estilo estaría indicando una aparente homogeneidad estilística quebradeña, las nuevas líneas de evidencia relacionadas con los estudios tecnológicos han ido aportando información sobre las sutiles maneras locales de hacer dentro de la Quebrada de Humahuaca.

Al Estilo Negro sobre Rojo lo acompaña otro estilo que ha sido denominado Estilo Negro y Blanco sobre Rojo o Tricolor (entre otras denominaciones, ver Marte et al. en este Boletín). No obstante, hacia finales del Período de Desarrollos Regionales la variedad tricolor es menos frecuente, mientras que el Estilo Negro sobre Rojo continúa teniendo una amplia distribución en la Quebrada de Humahuaca con algunas variables, tal como se describió con anterioridad (Nielsen 1997, 2001, 2010; Cremonte 2006).

Los motivos o diseños representados en las piezas cerámicas Estilo Negro sobre Rojo corresponden a bandas reticuladas, triángulos negros o reticulados, espirales, "manos o alas", cruces, dameros, entre los principales, los cuales están organizados en diferentes configuraciones (figs. 1a, b y c). Las decoraciones pueden aparecer tanto en el interior de pucos como en el exterior de cántaros, ollas y, además, en bordes de diversas piezas (Nielsen 1997, 2001; Palma 1998; Cremonte 2006).

Teniendo en cuenta todo lo anteriormente mencionado, el objetivo de este trabajo es aportar información a la problemática relacionada con la tecnología cerámica de la región, contribuyendo con resultados sobre las composiciones pigmentarias utilizadas en el Estilo Negro sobre Rojo. Consideramos que a partir de estos y de otros datos aportados por investigadores de la región podemos plantear algunas hipótesis, que deberán seguir siendo revisadas, acerca de las mezclas pigmentarias y del proceso térmico usado en la realización de este tipo de decoraciones. Para ello se han empleado -junto con las técnicas tradicionales de microscopia óptica y difracción de rayos X (DRX)- la espectroscopia Raman (RS) y la microscopia electrónica de barrido con espectroscopia de energía dispersiva (MEB-EDS). Además, se tuvo en cuenta la vinculación entre los resultados de las diversas técnicas aplicadas con el fin de profundizar en las ventajas y las limitaciones de las mismas, como así también en los tipos de muestreo utilizados. 

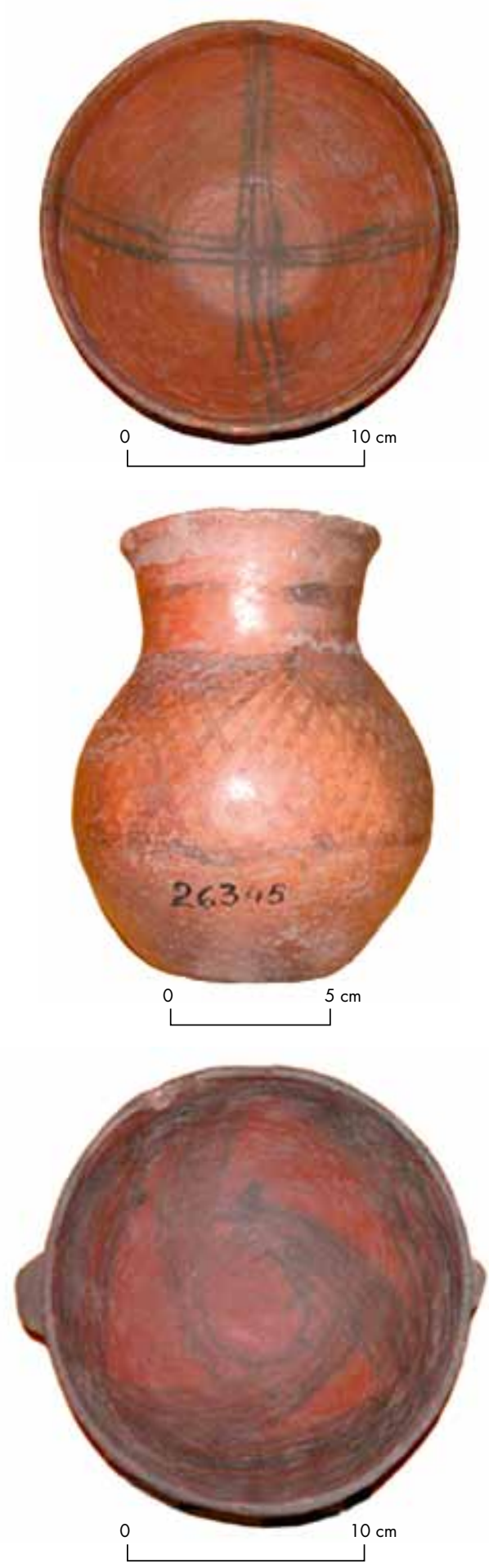

b)

Figura 1. a), b) y c). Piezas representativas del Estilo Negro sobre Rojo. Procedencia: Pukara de Tilcara, Quebrada de Humahuaca, colección Museo Etnográfico Juan B. Ambrosetti, Buenos Aires, Argentina.

Figure 1. a), b) \& c). Pieces representative of the Black on Red Style. Provenance: Pukara de Tilcara, Quebrada de Humahuaca. From the collection of Museo Etnográfico Juan B. Ambrosetti, Buenos Aires, Argentina.

\section{ANTECEDENTES}

En un trabajo de revisión (López \& Fiore 2010) hemos observado que en el Noroeste Argentino (NOA) se registra un desarrollo disímil en los análisis de las composiciones pigmentarias procedentes de distintos contextos arqueológicos y tipos de muestras. Esto es debido a diversos factores, como el desigual acceso a las técnicas analíticas y, fundamentalmente, la distinta formación de los arqueólogos de la región de estudios, entre otros. Por ello es de esperar que la implementación de técnicas analíticas específicas y la programación de trabajos interdisciplinarios de manera más sistemática produzcan un aporte de resultados vinculados a las diferentes tecnologías utilizadas por las diversas sociedades en el pasado.

En este sentido, los trabajos precedentes constituyen ejemplos de distinto alcance en cuanto al potencial de las técnicas analíticas aplicadas al estudio de muestras tomadas sobre materiales cerámicos (engobes y pinturas), así como sobre sus residuos (pigmentos o mezclas pigmentarias) hallados en contextos arqueológicos de distinta naturaleza. Entre las técnicas aplicadas habitualmente hemos encontrado la de DRX y la de MEB-EDS (Botto et al. 1998; Cremonte et al. 2003; López 2004, 2007; De la Fuente 2005; De la Fuente et al. 2007; De la Fuente \& Martínez 2008; De la Fuente et al. 2009; Cremonte \& Botto 2009).

Entre los ejemplos más interesantes relevados en el trabajo de revisión de López y Fiore (2010) podemos citar aquellos que, a partir de la implementación de estas y otras técnicas, han permitido avances importantes no solo en la comprensión de las características físicas y químicas de las mezclas pigmentarias propiamente dichas, sino que también en: a) la comprensión de aspectos técnicos de su preparación, aplicación y uso (Cremonte \& Botto 2009); b) en aspectos vinculados a la manufactura cerámica, como la determinación de la temperatura de cocción (Cremonte et al. 2003; Cremonte \& Botto 2009) y c) en la generación de nuevas agrupaciones arqueológicas basadas en criterios tecnológicos (Cremonte et al. 2003; López 2004, 2007). Estas últimas son las que permiten al arqueólogo superar los clásicos ordenamientos tradicionales, en particular aquellos basados en "estilos" cerámicos de amplio rango cronológico y distribución regional -como el caso del Negro sobre Rojo- que volvían poco clara su procedencia (Cremonte et al. 2003; López 2007; Palamarczuk 2011). Afortunadamente en la última década el énfasis puesto en este tipo de análisis ha sido tan importante que, incluso, existen proyectos en marcha tendientes a crear bases de datos de pigmentos arqueológicos (De la Fuente et al. 2009). 
En lo que concierne en particular al color negro empleado en cerámica Estilo Negro sobre Rojo, podemos decir que, en líneas generales, este puede deberse a distintos orígenes: mineral, orgánico o a una combinatoria de ambos. Cuando el origen es mineral, se trata de compuestos fundamentalmente de hierro y manganeso (Botto et al. 1998; López 2004; De la Fuente 2005; De la Fuente et al. 2007; De la Fuente \& Martínez 2008; Acevedo 2011). Por ejemplo, en lo que respecta a los compuestos de hierro suele plantearse que cuando la hematita $\left(\mathrm{Fe}_{2} \mathrm{O}_{3}\right)$ se encuentra en condiciones de cocción con bajo contenido de oxígeno se transforma en magnetita $\left(\mathrm{Fe}_{3} \mathrm{O}_{4}\right)$ (Botto et al. 1998; López 2004). Bajo esta hipótesis de trabajo el tipo de atmósfera de cocción y el manejo de las temperaturas de las piezas resultaría determinante en el proceso del color. En este sentido encontramos que para el estilo en cuestión se ha planteado la posibilidad de que los pigmentos negros asignados al compuesto magnetita $\left(\mathrm{Fe}_{3} \mathrm{O}_{4}\right)$ sobre un engobe color rojo podrían haberse logrado mediante la bicocción de las piezas (Botto et al. 1998).

En cuanto al manganeso, encontramos que este puede aparecer en distintos minerales: jacobsita $\left(\mathrm{MnFe}_{2} \mathrm{O}_{4}\right)$ cuando fue sometido a temperaturas del orden de 900$1000^{\circ} \mathrm{C}$ (Centeno et al. 2012); psilomelano $\left[(\mathrm{Ba}, \mathrm{Mn})_{3}(\mathrm{O}\right.$, $\left.\mathrm{OH})_{6} \mathrm{Mn}_{8} \mathrm{O}_{16}\right)$ ], cuando pasó por un proceso térmico de al menos $800^{\circ} \mathrm{C}$ (De la Fuente et al. 2007 , De La Fuente \& Martínez 2008); bixbyta $\left[(\mathrm{Mn}, \mathrm{Fe})_{2} \mathrm{O}_{3}\right]$, cuando pasó por temperaturas de alrededor de $450^{\circ} \mathrm{C}$ (Schweizer \& Rinuy 1982) o pirolusita $\left(\mathrm{MnO}_{2}\right)$, indicando que no hubo proceso térmico alguno (De la Fuente \& Martínez 2008).

En los casos en que se ha registrado el uso de carbón (de origen mineral u orgánico), generalmente este ha sido utilizado solo o combinado con óxido de hierro en estado reducido (magnetita $\left(\mathrm{Fe}_{3} \mathrm{O}_{4}\right.$ ), lo cual contribuiría con el color negro (Botto et al. 1998; Acevedo 2011).

En términos generales se ha formulado que el color rojo logrado en piezas cerámicas del Estilo Negro sobre Rojo se encuentra relacionado con un engobe rico en hierro, donde el componente hematita $\left(\mathrm{Fe}_{2} \mathrm{O}_{3}\right)$, sería el responsable del color (Botto et al. 1998).

En síntesis, la técnica utilizada por los alfareros en la aplicación del diseño en piezas cerámicas Estilo Negro sobre Rojo ha llevado a postular distintas hipótesis. Por ejemplo, la bicocción o la utilización de manganeso como pigmento para lograr el color negro sobre un engobe rojo (Botto et al. 1998; López 2004). Sin embargo, sobre este tema en particular se sigue avanzando en la investigación mediante la implementación de técnicas arqueométricas, para afirmar algunas de las hipótesis planteadas para la tecnología cerámica usada en la Quebrada de Humahuaca.

\section{MATERIALES Y MÉTODOS}

\section{Las muestras}

Los fragmentos cerámicos con decoración negro sobre rojo aquí analizados ( $\mathrm{N}: 5$ sobre un total de 50) fueron extraídos por excavación arqueológica en el sitio Pintoscayoc 1 o Alero de las Circunferencias, ubicado en el norte de la Quebrada de Humahuaca, Argentina (fig. 2). El sitio se encuentra ubicado dentro de la localidad de Pintoscayoc, emplazada en las nacientes del río Grande, entre las actuales localidades de Azul Pampa e Iturbe a $3646 \mathrm{msnm}$. La ubicación corresponde al tramo superior de dicha quebrada, lo que ha sido denominado escalón altitudinal de "quebradas altas", región que se sitúa entre los 3200 y los 4600 msnm (Hernández Llosas 1998, 2000, 2001, 2006).

Los fragmentos cerámicos fueron extraídos de la capa $51^{\mathrm{a}}$ y $2^{\mathrm{a}}$ extracción (parte de la capa 5 cumbre). Estos fueron adscritos cronológicamente sobre la base de un fechado radiocarbónico contextual realizado sobre carbón vegetal, que dio como resultado un fe-

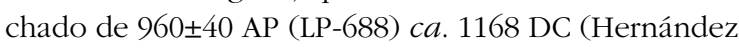
Llosas 1998). El material estudiado se ubica, entonces, a finales del segmento cronológico conocido para la Quebrada de Humahuaca como Período de Desarrollos Regionales I (PDRI), que se despliega entre $c a$. 900-1250 DC (Nielsen 1997, 2010).

Los fragmentos que fueron seleccionados para muestrear poseen en su superficie decoración del Estilo Negro sobre Rojo, específicamente, diseños de reticulados, aunque estos están organizados en diferentes configuraciones, no pudiéndose establecer qué diseño en particular conserva dentro de los denominados reticulados. Los mismos no han podido ser relacionados con alguna forma de vasija determinada, debido a las características de tamaño $(2,50 \times 3,00 \mathrm{~cm})$ y forma de los fragmentos.

Según el análisis petrográfico realizado a estas muestras podemos decir que poseen las siguientes características petrográficas: a) una cantidad moderada a muy abundante de líticos metamórficos, esquistos/esquistos micáceos y filitas, tamaño grueso a muy grueso; b) cantidad muy abundante de cuarzos redondeados; c) cantidad de plagioclasas muy abundantes de tamaño fino a medio y d) presencia de anfíboles. Estas coinciden con las características de la región en estudio, es decir, serían de origen local (Acevedo 2011).

Por las características mencionadas consideramos que estos fragmentos fueron propicios para aplicar las técnicas analíticas que se describen en este trabajo. De los cinco fragmentos seleccionados como muestras, en 


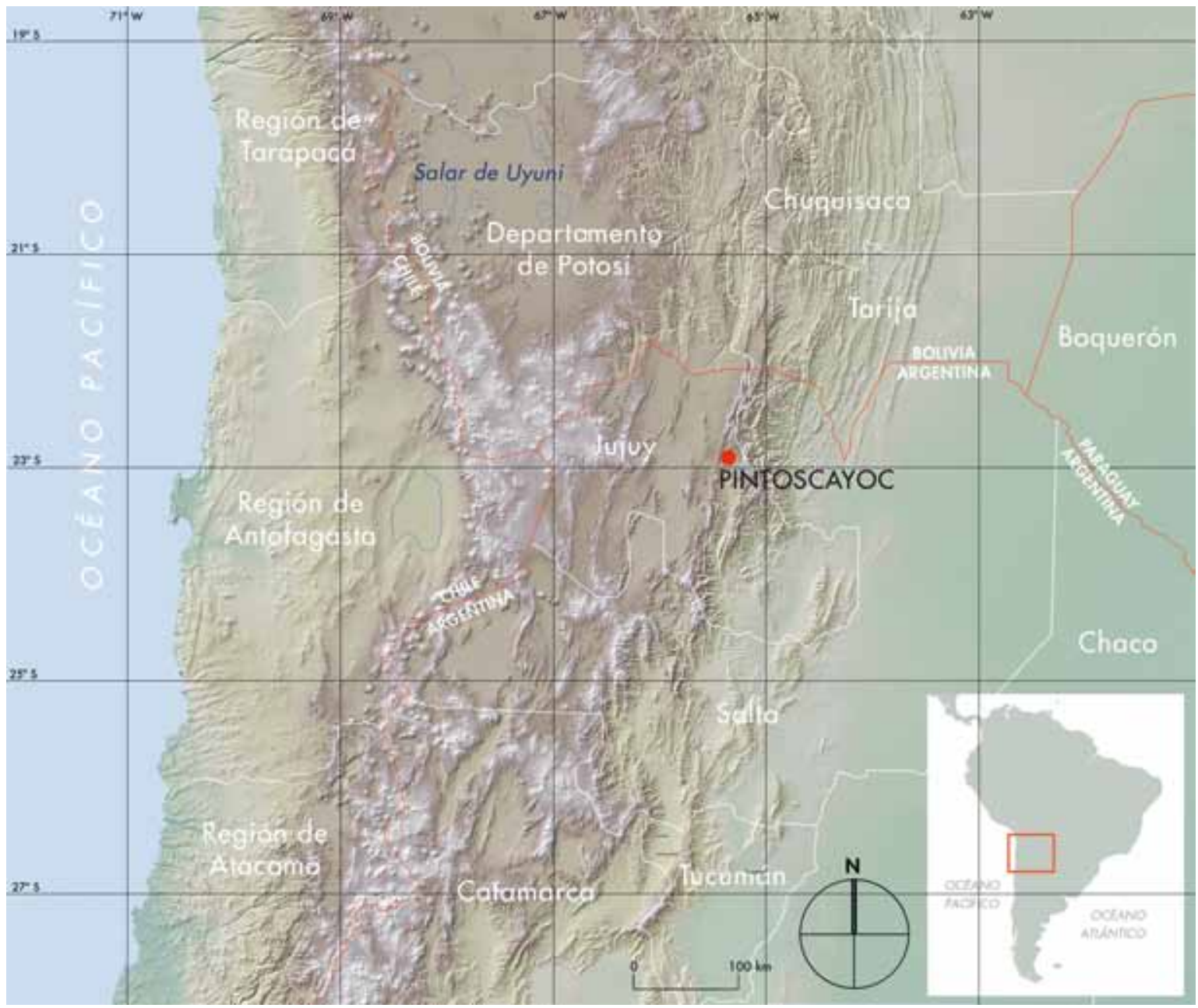

Figura 2. Localización de la localidad de Pintoscayoc, Quebrada de Humahuaca, Jujuy, Argentina. Figure 2. Location of the locality of Pintoscayoc, Quebrada de Humabuaca, Jujuy, Argentina.

tres de ellos (5, 7 y 9) se analizó el pigmento rojizomorado, mientras que en las otras dos muestras restantes (6 y 8) se estudió el pigmento negro (fig. 3). Para los estudios de caracterización de pigmentos presentes en todas estas muestras se emplearon dos metodologías de trabajo distintas: análisis de polvos y de fragmentos. De manera preliminar, y respetando el modo clásico de adquisición de muestras de pigmentos, se analizaron los polvos obtenidos del raspado de las superficies de los fragmentos. Luego, se analizaron directamente las superficies de los fragmentos cerámicos, sin ninguna preparación previa. Es decir, de los cinco fragmentos que constituyen la muestra original se analizaron un total de diez tipos de muestras obtenidas de dos maneras diferentes, una semidestructiva (polvos) y otra no destructiva, ya que implicó la "lectura" directa sobre el fragmento.

\section{Las técnicas}

Las muestras seleccionadas fueron analizadas mediante las técnicas de microscopia óptica (MO) en el laboratorio del IDA (Instituto de Arqueología de la Facultad de Filosofía y Letras, Universidad de Buenos Aires), microscopia electrónica de barrido con espectroscopia de energía dispersiva (MEB-EDS), difracción de rayos X (DRX) y espectroscopia Raman (RS) en laboratorios de la Comisión Nacional de Energía Atómica de Buenos Aires.

La caracterización por MO se efectuó mediante un microscopio simple o lupa trinocular Arcano modelo ZTX1:4 equipado con una cámara Motic modelo Moticam 352 , con aumentos comprendidos entre 10x y 80x.

El análisis elemental de las muestras en polvo se realizó por MEB-EDS empleando un equipo Philips 515 
a)

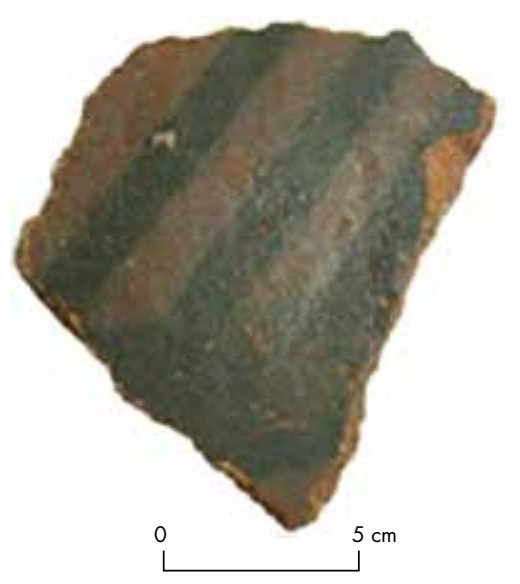

b)

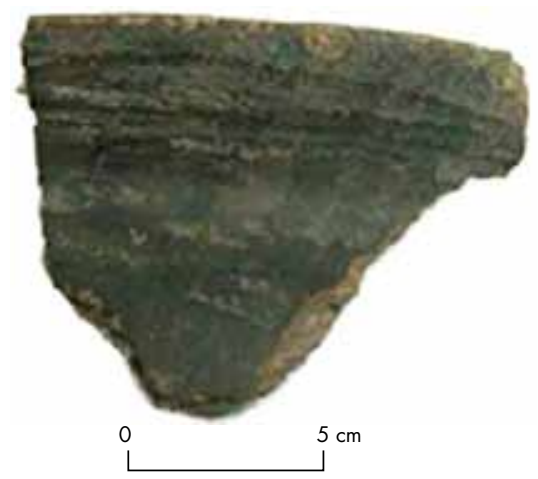

Figura 3. Fotografías de algunos de los fragmentos analizados: a) frag. $\left.\mathrm{N}^{\circ} 6, \mathrm{~b}\right)$ frag. $\mathrm{N}^{\circ} 8$.

Figure 3. Photographs of some of the fragments analyzed: a) frag. 6 , b) frag. 8 .

microscope (Philips Export B.V., Eindhoven, The 134 Netherlands) equipado con sonda EDS EDX PV9100 probe (EDX International Inc., Prairie, View, 135 IL, USA). Todos los espectros fueron obtenidos a $20 \mathrm{kV}$ y con un tiempo de análisis de 40s. La composición elemental, la estructura superficial y la morfología fueron analizadas para cada partícula observada y compilada para cada muestra. Cabe destacar que no fue necesaria la metalización de las muestras, ya que se depositaron sobre una cinta conductora de carbono y se analizaron directamente. Se observaron y fotografiaron a distintos aumentos y se hizo el análisis elemental sobre diferentes partes de las muestras. Este método de análisis permite determinar elementos cuyo peso atómico sea mayor que cinco.

La técnica de DRX fue utilizada para la identificación de las fases cristalinas presentes en todas las muestras analizadas. El análisis se realizó a temperatura ambiente empleando un equipo Philips PW 3710 con monocromador y radiación de $\mathrm{Cu}(\mathrm{K}-\mathrm{Alph} 1[\AA]=1.54060)$. Se utilizó un paso de 0.02 en $2 \theta$ con un tiempo de contaje de dos segundos por paso y con un generador de $30 \mathrm{~mA}$,
$40 \mathrm{kV}$. Se obtuvo registro en el rango de $10^{\circ}<2 \theta<70^{\circ}$, zona suficiente para la tarea de identificación y caracterización de la muestra. El difractograma obtenido fue procesado con el conjunto de programas PC-APD de Philips que permite obtener la posición y la intensidad de los picos del diagrama. Para la identificación de fases presentes se empleó un programa denominado PC-Identify y la base de datos de la ICDD.

Los espectros RS fueron adquiridos en un espectrómetro comercial LabRAM HR (Horiba Jobin Yvon) equipado con doble monocromador y detector CCD (Charge Coupled Device) de resolución espectral de $1.2 \mathrm{~cm}^{-1}$. Se utilizó la línea $514.5 \mathrm{~nm}$ de un láser de argón como fuente excitadora y la potencia del láser sobre la muestra se mantuvo por debajo de $0.2 \mathrm{~mW}$ de modo de evitar el calentamiento del punto a observar. El microscopio acoplado al espectrómetro (objetivos de $\mathrm{x} 10, \mathrm{x} 50, \mathrm{x} 100)$ permite concentrar el haz en una región de estudio aproximadamente circular de $50 \mu \mathrm{m}^{2}$. El tiempo de adquisición y el número de espectros promediados fue ajustado en cada punto siendo en general del orden de $60 \mathrm{~s}$ de adquisición y cinco promedios para cada espectro.

Ninguna de las técnicas empleadas requiere una preparación especial de las muestras, con el beneficio adicional de que las mismas pueden ser utilizadas para análisis complementarios.

\section{RESULTADOS}

Para analizar correctamente los resultados, es importante considerar cómo estos se ven afectados por el tipo de muestra y la forma de muestreo. Estas consideraciones tienen distintos efectos en cada técnica. En la técnica de DRX el área analizada es del orden de $1 \mathrm{~cm}^{2}$, mientras que en la técnica de RS se analizan superficies circulares del orden de $10 \mu \mathrm{m}$ de diámetro (aunque puede llegarse al orden del micrón) en las condiciones de Raman; es importante en muestras no homogéneas, como las arqueológicas, estudiar varios puntos. Para el análisis de las muestras involucradas en este trabajo se midieron del orden de 5-10 puntos en cada muestra.

Los cinco fragmentos analizados por microscopia óptica revelan la presencia de color rojizo-morado como engobe de base y por sobre el engobe diseños en líneas negras (figs. $4 \mathrm{a}$ y b). Ambos colores y tratamientos presentan el mismo tipo de carga matérica. En algunos casos, las líneas negras no están perfectamente delineadas, sino que parecen esfumarse sobre el rojizo-morado del engobe de base; esto pudo ser observado a 80x de aumento (fig. 4c). No se pudieron distinguir líneas de 

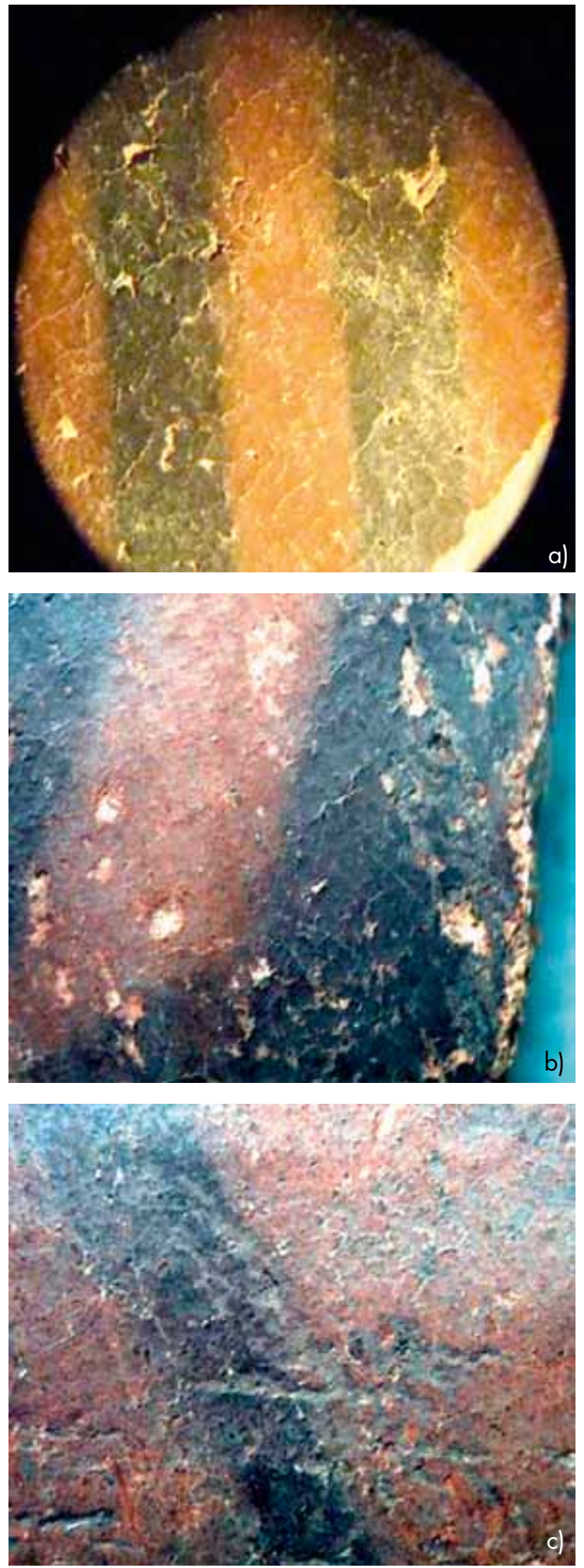

Figura 4. Micrografías Negro sobre Rojo: a) 20x, b) 40x, c) 80x (frag. $\mathrm{N}^{\circ}$ 6).

Figure 4. Micrographs of Black on Red pigments: a) 20x, b) 40x c) $80 x$ (frag. 6). alisado, aunque la regularidad de las superficies permite suponer que estuvieron alisadas antes de la aplicación del engobe. Tampoco se observaron líneas de pulido ni rastros de uso.

Con relación al tipo de cocción de los fragmentos cerámicos en estudio, se registró el uso de una atmósfera de cocción oxidante, lo que fue observado en las pastas. No se observaron núcleos de cocción que permitieran hacer inferencias sobre el uso de una atmósfera de cocción reductora completa, una atmósfera oxidante incompleta con márgenes de núcleos rectos o una atmósfera oxidante incompleta con márgenes de núcleos difusos (López 1999-2001).

El análisis de MEB-EDS se realizó sobre muestras en polvo de color negro provenientes del raspado de las superficies externas pintadas de los fragmentos, para analizar la composición elemental. En las muestras analizadas se detectó presencia de aluminio (Al), carbono $(\mathrm{C})$, calcio $(\mathrm{Ca})$, cobre $(\mathrm{Cu})$, hierro $(\mathrm{Fe})$, potasio $(\mathrm{K})$, magnesio $(\mathrm{Mg})$, sodio $(\mathrm{Na})$, oxígeno $(\mathrm{O})$ y silicio $(\mathrm{Si})$. No se detectó presencia de manganeso en ninguna de las muestras analizadas.

El análisis por DRX y RS de todas las muestras, tanto en polvo como sobre superficie de fragmentos, revela que los compuestos presentes en todas las muestras son bastante similares y los resultados obtenidos a partir de las técnicas utilizadas están en concordancia. En todos los casos se observó cuarzo $\left(\mathrm{SiO}_{2}\right)$ y carbón (C) en forma de grafito microcristalino, y también compuestos como dióxidos de titanio $\left(\mathrm{TiO}_{2}\right)$ en las fases de anatasa, rutilo o brukita junto con varios aluminosilicatos, lo cual es esperable en mezclas pigmentarias de materiales arcillosos. De hecho, estos compuestos han sido observados en zonas exteriores no coloreadas de las piezas tanto como en el interior de las mismas.

Los compuestos encontrados en ambos muestreos (polvo y fragmentos) coinciden en DRX y RS. Sin embargo, las muestras en polvo suelen mostrar más variedad de componentes relacionados con los materiales arcillosos, como cuarzo, anatasa y aluminosilicatos, generalmente presentes en la arcilla. Aparte, en el análisis de los fragmentos se identifican más claramente los compuestos responsables del color. A modo de ejemplo, en la figura 5 se presentan los espectros Raman obtenidos en distintos puntos del polvo (fig. 5a) y fragmento (fig. 5b) de la muestra $\mathrm{N}^{\circ} 6$. En los resultados obtenidos sobre fragmentos se detecta la presencia de magnetita $\left(\mathrm{Fe}_{3} \mathrm{O}_{4}\right)$ y grafito microcristalino como responsables del color negro, mientras que en los resultados sobre polvo se observa también cuarzo, anatasa, calcita $\left(\mathrm{CaCO}_{3}\right)$ y hematita $\left(\mathrm{Fe}_{2} \mathrm{O}_{3}\right)$. 

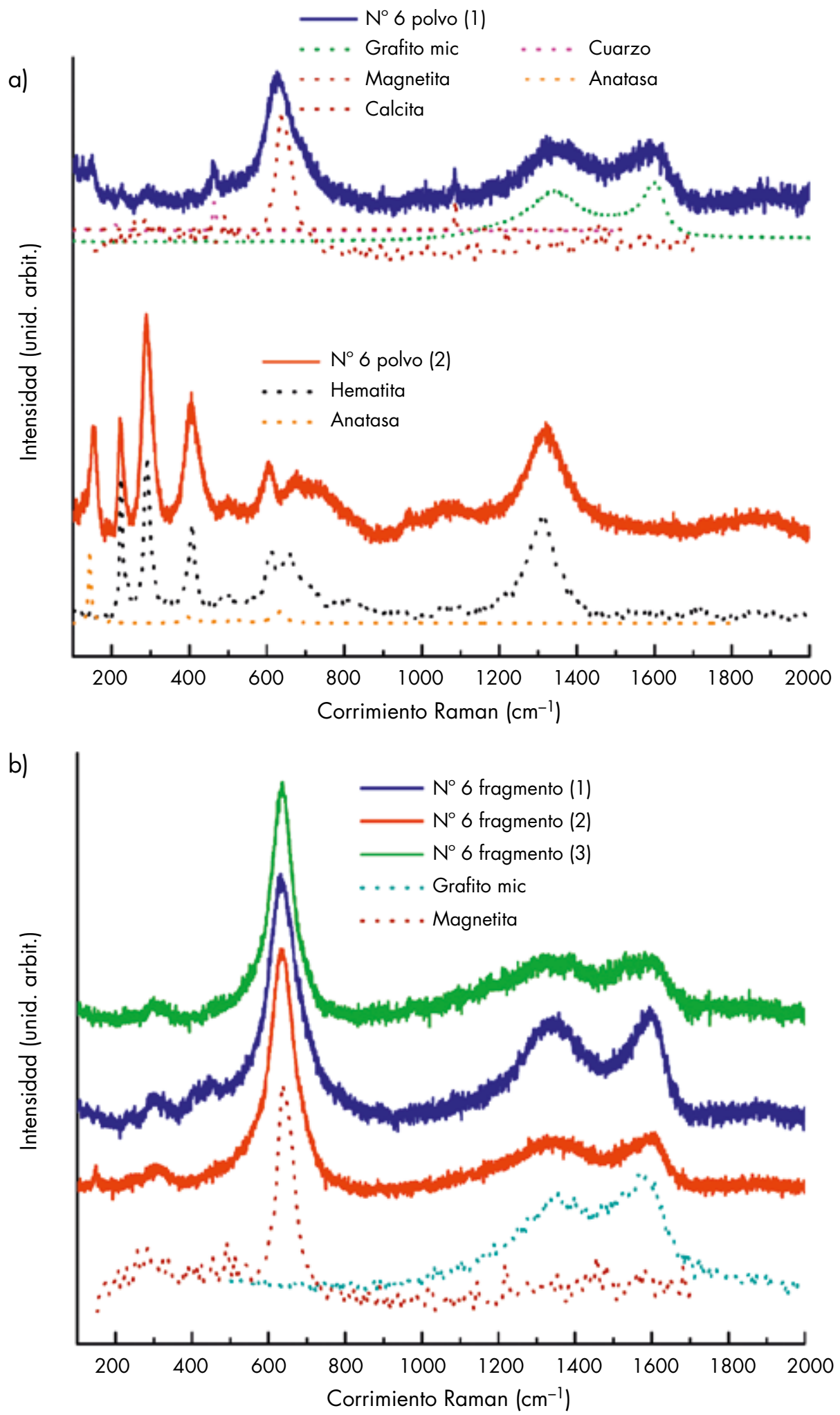

Figura 5. Espectros Raman obtenidos en distintos puntos de la muestra $\mathrm{N}^{\circ} 6$ para: a) polvo y b) fragmento. Se incluyen espectros de referencia en línea punteada.

Figure 5. Raman spectrographs obtained at different points of sample 6 for: a) dust and b) fragment. Dotted line indicates reference spectra. 
En los resultados de los análisis en RS, en general, tanto en muestras en polvo como en fragmentos, se observaron distintas fases del dióxido de titanio. Si bien con frecuencia aparece en su fase anatasa, alguna vez se observa, también, en la fase brukita y en muy pocas oportunidades en su fase rutilo. Sin embargo, en los resultados de los análisis de DRX, tanto en fragmentos como en polvos, generalmente aparece el dióxido de titanio en su fase de rutilo. Lo interesante es que el dióxido de titanio en sus fases anatasa y brukita es posible de encontrar a bajas temperaturas y hasta los $600^{\circ} \mathrm{C}, \mathrm{y}$ a partir de los $800^{\circ} \mathrm{C}$ aproximadamente se transforma en rutilo (Czanderna et al. 1958). Esto podría ser un indicio para sospechar el uso de bajas temperaturas en la cocción de las piezas cerámicas, característica que registran otros autores para la cerámica de Quebrada de Humahuaca (Botto et al 1998).

En la Tabla 1 se presentan los resultados obtenidos por las técnicas de DRX y RS sobre las superficies de fragmentos Negro sobre Rojo. Debe destacarse que las técnicas empleadas no permiten la cuantificación de los compuestos reportados. Sin embargo, estos son presentados en la tabla de acuerdo con el puntaje asignado por el programa de identificación. Además, se han excluido los componentes atribuidos a la matriz.

Tabla 1. Resultados de análisis sobre superficies externas de fragmentos.

Table 1. Results of the analysis of external surfaces of the fragments.

\begin{tabular}{|c|c|c|}
\hline Muestras & $\begin{array}{c}\text { Raman } \\
\text { compuestos }\end{array}$ & $\begin{array}{c}\text { DRX } \\
\text { compuestos }\end{array}$ \\
\hline $\mathrm{N}^{\circ} 5$ Rojizo-morado & $\begin{array}{l}\text { Hematita } \\
\text { Grafito mic } \\
\text { Magnetita }\end{array}$ & $\begin{array}{l}\text { Hematita } \\
\text { Grafito } \\
\text { Magnetita }\end{array}$ \\
\hline $\mathrm{N}^{\circ} 6$ Negro & $\begin{array}{l}\text { Magnetita } \\
\text { Grafito mic }\end{array}$ & $\begin{array}{c}\text { Grafito } \\
\text { Magnetita } \\
\text { Hematita }\end{array}$ \\
\hline $\mathrm{N}^{\circ} 7$ Rojizo-morado & $\begin{array}{l}\text { Hematita } \\
\text { Grafito mic }\end{array}$ & $\begin{array}{l}\text { Hematita } \\
\text { Grafito } \\
\text { Magnetita }\end{array}$ \\
\hline $\mathrm{N}^{\circ} 8$ Negro & $\begin{array}{l}\text { Grafito mic } \\
\text { Magnetita }\end{array}$ & $\begin{array}{c}\text { Grafito } \\
\text { Magnetita } \\
\text { Hematita }\end{array}$ \\
\hline $\mathrm{N}^{\circ} 9$ Rojizo-morado & $\begin{array}{l}\text { Hematita } \\
\text { Grafito mic }\end{array}$ & $\begin{array}{c}\text { Hematita } \\
\text { Magnetita } \\
\text { Grafito }\end{array}$ \\
\hline
\end{tabular}

Se ha observado hematita en todas las muestras de engobe rojizo-morado por ambas técnicas empleadas para la identificación (fig. 6), aunque se han detectado también grafito y otros compuestos relacionados con materiales arcillosos. La contribución de estos últimos es más notoria en la técnica de DRX, ya que la superficie analizada es mayor. Por último, en las muestras 6 y 8 el origen del color negro se ha asociado a la presencia de magnetita y carbón (fig. 7) y en ningún caso se detectaron compuestos de manganeso como responsables del color negro, en concordancia con los resultados de SEM-EDX.

\section{CONCLUSIONES}

En este trabajo hemos expuesto el beneficio de trabajar con distintas técnicas analíticas de manera combinada con el fin de aportar a una problemática arqueológica. Asimismo, hemos podido comparar las ventajas y las limitaciones encontradas según la forma de muestreo: polvo y fragmento, siendo este último formato no solo más beneficioso en torno a la claridad de los resultados observados en relación con este tema, sino también en relación con la conservación de las muestras.

En cuanto a la problemática de la tecnología del color en materiales cerámicos, los resultados presentados asocian el color rojo al contenido de óxidos de hierro, en concordancia con estudios previos para la Quebrada de Humahuaca y el NOA en general (Botto 1998; López 2004, 2007; De la Fuente \& Martínez 2008; Acevedo 2011). En relación con el color negro, nuestros resultados lo asocian con la presencia de magnetita y grafito microcristalino y en ningún caso con la presencia de compuestos de manganeso.

Acerca del origen de los pigmentos, pensamos que es posible hallar magnetita en sectores restringidos como fuente natural de recursos en la Quebrada de Humahuaca (Turner 1964), aunque también es factible preparar magnetita tratando hematita térmicamente en condiciones reductoras (Shepard 1985). Con lo cual, cualquiera de las dos opciones tecnológicas es útil para la preparación de las mezclas pigmentarias de color negro que pudieron ser utilizadas en la decoración de vasijas Estilo Negro sobre Rojo.

Hasta el momento, en la tecnología empleada para la producción de las piezas Negro sobre Rojo, dos son las hipótesis que se han postulado para este tipo de decoración: monococción en condiciones de temperatura y atmósfera controladas (Striova et al. 2006; Iordanis \& García-Guinea 2011) y bicocción (Botto et al. 1998). En el primer caso, la cocción sería en atmósfera oxidante 

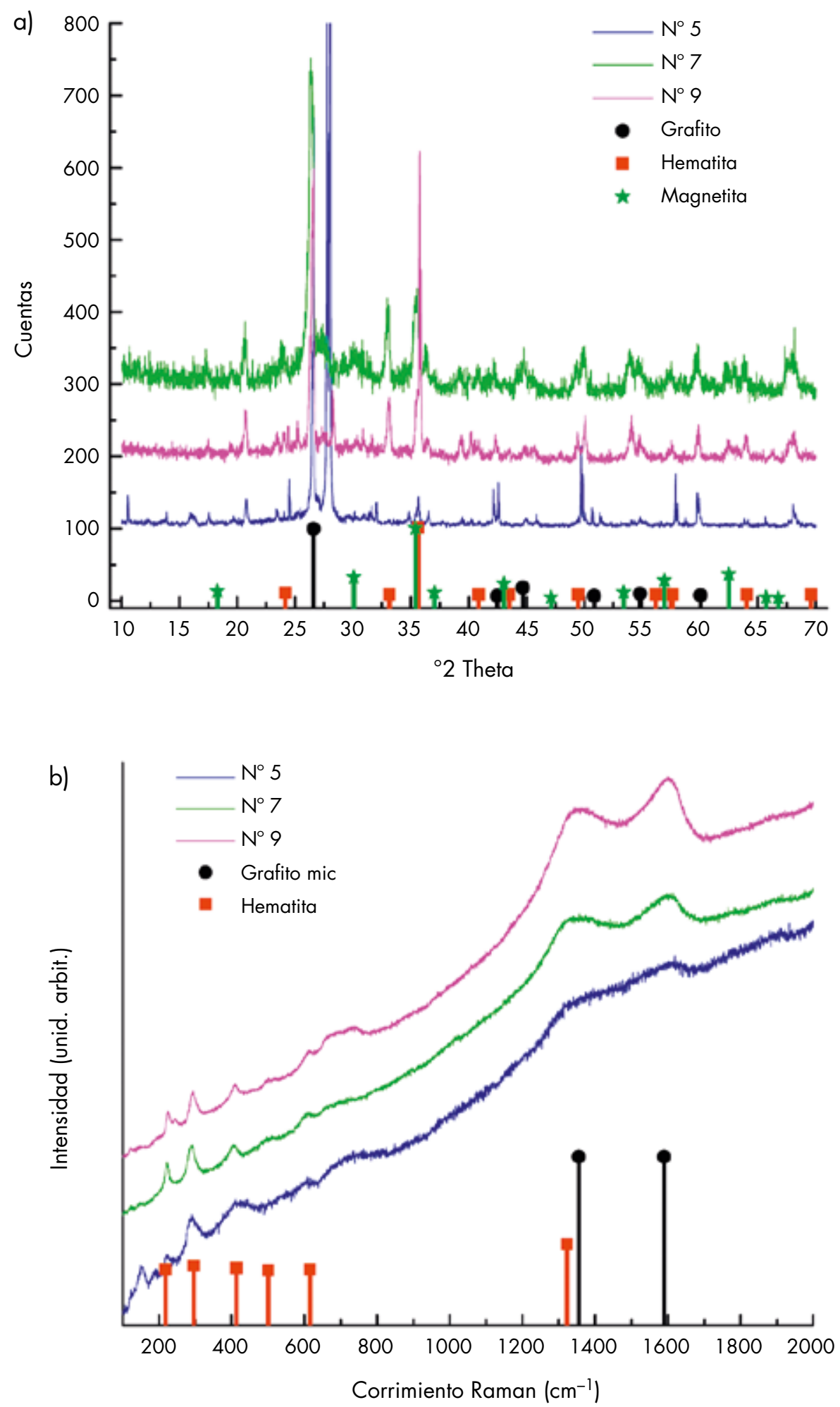

Figura 6. Resultados obtenidos para muestras con pigmentos rojos: a) difractograma de rayos X y b) espectros Raman. Figure 6. Results obtained for samples with red pigment: a) X-ray diffraction and b) Raman spectra. 

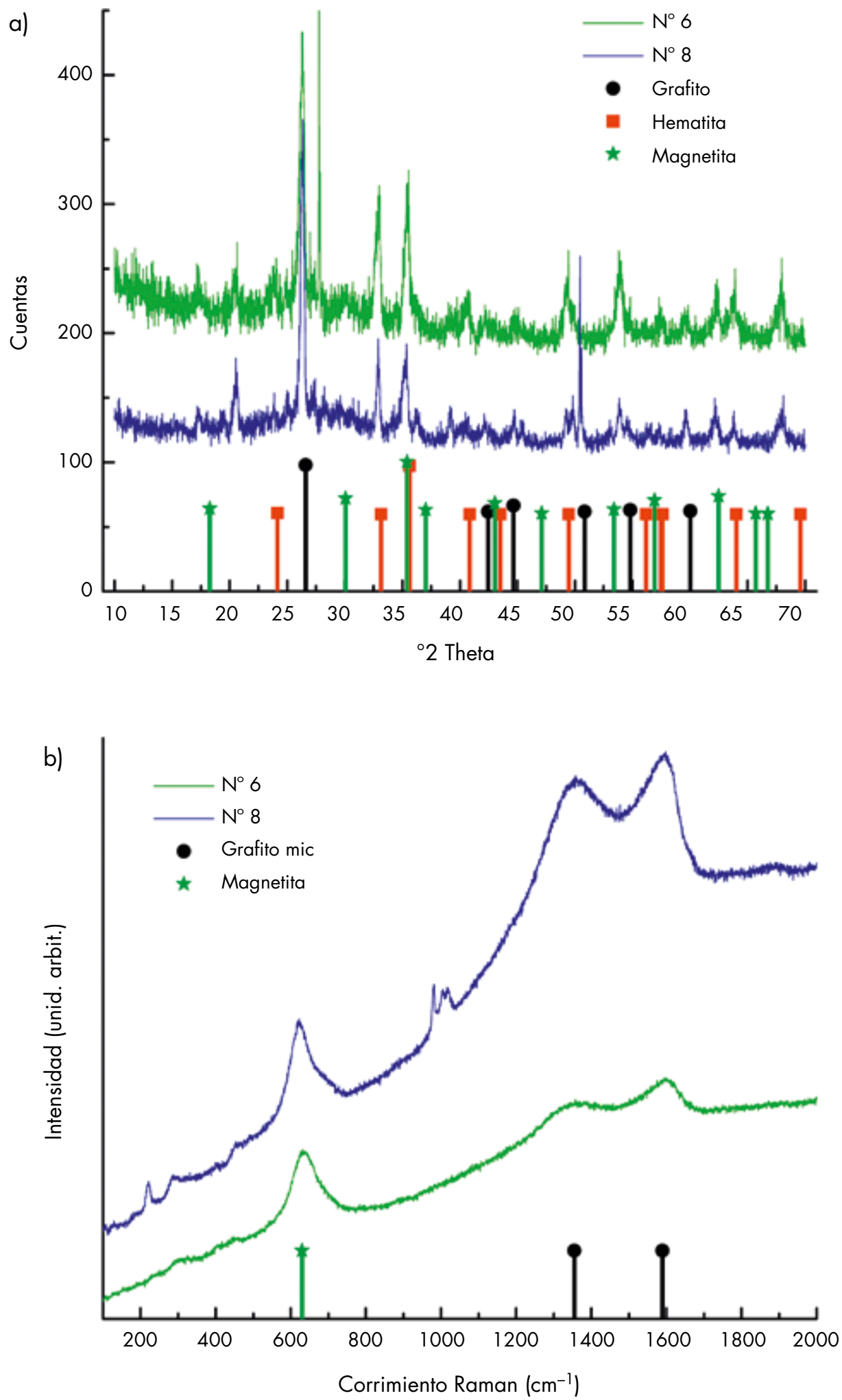

Figura 7. Resultados obtenidos para muestras con pigmentos negros: a) difractograma de rayos X y b) espectros Raman. Figure 7. Results obtained for samples with black pigment: a) X-ray diffractogram and b) Raman spectra. 
bajo un control de temperatura muy cuidadoso, evitando la transformación de magnetita a hematita y dando lugar a un fondo rojo mientras se mantiene la decoración negra. En el segundo caso, se consideran dos etapas de cocción: una oxidante $\left(\sim 500^{\circ} \mathrm{C}-600^{\circ} \mathrm{C}\right)$ y luego una reductora a temperaturas mayores $\left(\sim 700^{\circ} \mathrm{C}\right)$.

Considerando los resultados expuestos, tanto de las muestras estudiadas en este trabajo como los resultados obtenidos de trabajos previos de otros investigadores, y con relación a la tecnología empleada para la producción de las piezas cerámicas Estilo Negro sobre Rojo, la presencia de magnetita y carbón sugeriría el empleo de una cocción en atmósfera reductora. Sin embargo, las observaciones en microscopia óptica de la pasta cerámica de los fragmentos estudiados y de los fragmentos que conforman la muestra cerámica ubicada hacia finales del Período de Desarrollos Regionales I de Pintoscayoc 1 (Acevedo 2011), indican una cocción de las piezas en atmósfera oxidante, sin evidencia de núcleos de cocción que revelen la posible utilización de una atmósfera escasa en oxígeno o diferentes pasos en la cocción de las piezas cerámicas. No obstante, las evidencias de la bicocción en los fragmentos cerámicos podrían ser sutiles debido al uso de bajas temperaturas que solo afectaran en un último momento la superficie cerámica.

Es posible, entonces, pensar en la posibilidad de obtener magnetita como materia prima dentro del área y/o la preparación previa de la misma en condiciones reductoras, y con esto poder disponer de la mezcla pigmentaria de color negro que se utilizaría en la decoración de las piezas cocidas en un solo evento y en condiciones oxidantes a bajas temperaturas. En efecto, de nuestros resultados no se desprenden indicios de una posible bicocción.

No obstante las hipótesis aquí presentadas, estos resultados constituyen una primera contribución a la problemática planteada, en la cual fue fundamental el uso de técnicas combinadas, el tipo de muestreo y el trabajo interdisciplinario. En este sentido, estos primeros resultados aportaron información sobre la composición de algunos de los pigmentos y las posibilidades del manejo de la tecnología cerámica que estaría desarrollándose en la región.

En síntesis, los diseños observados en los distintos tipos de piezas cerámicas del Estilo Negro sobre Rojo de la Quebrada de Humahuaca son muy similares entre sí, independientemente de su contexto o sitio de procedencia. Sin embargo, los análisis tecnológicos aplicados en estos últimos años han comenzado a mostrar variabilidad local (López 2004; Cremonte 2006; Acevedo 2011). En lo que se refiere estrictamente a la obtención del color, los resultados aquí presentados corroboran el uso de los mismos componentes del color ya anteriormente observados para el Período Desarrollos Regionales en la zona de Quebrada de Humahuaca (Botto et al. 1998), agregando nuevas hipótesis de tratamiento a la tecnología del color.

RECONOCIMIENTOS A la Dra. María Isabel Hernández Llosas, directora del proyecto Pintoscayoc, por permitirnos abordar el análisis del material cerámico desde una nueva perspectiva. Al Dr. Guillermo de la Fuente por la realización de los estudios petrográficos y sus comentarios sobre este trabajo. Asumimos que el contenido y las conclusiones del mismo son de nuestra responsabilidad.

\section{REFERENCIAS}

Acevedo, V. J., 2011. Tecnología, uso y consumo de los conjuntos cerámicos del Alero Pintoscayoc 1, Quebrada de Humahuaca, Jujuy. Tesis para optar al título de Licenciada en Ciencias Antropológicas con orientación Arqueológica. Facultad de Filosofía y Letras, Universidad de Buenos Aires, Argentina.

Botto, I. L.; V. L. Barone, M. B. Cremonte \& M. A. Sánchez, 1998. Estudios arqueométricos de cerámicas provenientes del Noroeste Argentino. Información tecnológica 9 (6): 79-86.

Centeno, S. A.; V. I. Williams, N. C. Little \& R. J. Speakman, 2012. Characterization of surface decorations in pre-Hispanic archaeological ceramics by Raman spectroscopy, FTIR, XRD and XRF. Vibrational Spectroscopy 58: 119-124.

Cremonte, M. B., 1985. Alcances y objetivos de los estudios tecnológicos en la cerámica arqueológica. Anales de Arqueología y Etnología 38/40: 179-217. Mendoza: Universidad Nacional de Cuyo.

— 1991. Análisis de muestras cerámicas de la Quebrada de Humahuaca. Avances en Arqueología 1: 17-42. Buenos Aires: Instituto Interdisciplinario de Tilcara, Universidad de Buenos Aires.

— 2006. El estudio de la cerámica en la reconstrucción de las historias locales. El sur de la Quebrada de Humahuaca (Jujuy, Argentina) durante los Desarrollos Regionales e Incaico. Chungara 38 (2): 239-247.

Cremonte, M. B.; M. Baldini \& I. L. Botto, 2003. Pastas y colores. Un camino al conocimiento del estilo Portezuelo de Aguada. Intersecciones en Antropología 4: 3-16.

Cremonte, M. B. \& I. L. Botto, 2009. Unas vasijas especiales de contextos tardíos del Noroeste Argentino. Manufactura de los "pucos bruñidos". Estudios Atacameños 37: 63-77.

Cremonte, M. B. \& M. F. Bugliani, 2010. Pasta, forma e iconografía. Estrategias para el estudio de la cerámica arqueológica. Xama 19-23: 239-262, Mendoza.

Czanderna, A. W.; C. N. Ramachandra Rao \& J. M. Honig, 1958. The anatase-rutile transition. Part 1.-Kinetics of the transformation of pure anatase. Transactions of the Faraday Society 54: 1069-1073.

De la Fuente, G. A., 2005. Technical Study of Pigments and Paintings in Archaeological Ceramics from Northwestern Argentine Region: An Archaeometric Approach and Implication for Their Conservation through SEM-EDS. Materials Research Society. Symposium Proceedings Vol. 852: 255-262.

De la Fuente, G. A.; N. Kristcautzky \& G. Toselli, 2007. Pigmentos, engobes y alfareros: Una aproximación arqueométrica (MEBEDS) al estudio de pigmentos en cerámicas arqueológicas del Noroeste Argentino: El caso del estilo cerámico "Aguada Portezuelo" del Valle de Catamarca. En Cerámicas arqueológicas. Perspectivas arqueométricas para su análisis e interpretación, B. Cremonte \& N. Ratto, Eds., pp. 39-47. San Salvador de Jujuy: Universidad Nacional de Jujuy.

De la Fuente, G. A. \& J. M. Martínez, 2008. Estudiando pinturas en cerámicas "Aguada Portezuelo" (ca. 600-900 AD) del 
Noroeste Argentino: Nuevos aportes a través de una aproximación arqueométrica por microespectroscopia Raman (MSR) Intersecciones en Antropología 9: 173-186.

De la Fuente, G. A.; A. C. Carreras, J. M. Pérez Martinez, S. Martín \& A. Riveros, 2009. Identificación y análisis de pigmentos y pinturas en cerámicas arqueológicas Sanagasta (ca. 900-1200 AD) e Inka (ca. 1480-1532 AD) a través de MEB-EDS, WD-EDS y microespectroscopia Raman (Abaucán, Tinogasta, Catamarca, Argentina). En Libro de resúmenes del III Congreso Argentino de Arqueometría y II Jornadas Nacionales para el estudio de Bienes Culturales, S. Bertolino, R. Cattáneo \& A. D. Izeta, Eds., p. 94, Córdoba.

Hernández Llosas, M. I., 1998. Pintoscayoc: Arqueología de las quebradas altas en Humahuaca. Tesis para optar al título de Doctor en Arqueología, Facultad de Filosofía y Letras Universidad de Buenos Aires, Argentina.

2000. Quebradas altas de Humahuaca a través del tiempo: El caso Pintoscayoc. Estudios sociales del NOA, año 3, $\mathrm{N}^{\circ} 2$. San Salvador de Jujuy: Instituto Interdisciplinario de Tilcara.

- 2001. Arte rupestre del Noroeste Argentino. Orígenes y contextos de producción. En Historia Argentina Prebispánica, Vol. 1, E. Berberián \& A. Nielsen, Eds., pp. 389-446. Córdoba: Editorial Brujas.

— 2006. Inkas y Españoles a la conquista simbólica del territorio de Humahuaca: Sitios, motivos rupestres y apropiación cultural del paisaje. Boletín del Museo de Arte Chileno Precolombino 11 (2): 9-34

IORDANIDIS A. \& J. GARCíA-GUINEA, 2011. A preliminary investigation of black, brown and red coloured potsherds from ancient upper Macedonia, northern Greece. Mediterranean Archaeology and Archaeometry 11 (1): 85-89.

López, M. A., 1999-2001. Los núcleos de cocción en las pastas cerámicas arqueológicas, indicadores y variables relacionados con algunos aspectos de la secuencia de producción. Xama 12-14: 133-149, Mendoza.

— 2004. Tecnología cerámica en La Huerta, Quebrada de Humahuaca, Provincia de Jujuy. Tesis para optar al título de Doctor en Arqueología, Facultad de Filosofía y Letras, Universidad de Buenos Aires.

- 2007. Complejidad social, especialización artesanal e innovaciones técnicas en Quebrada de Humahuaca: Un caso de cerámica inka Provincial? Arqueométricamente analizada. En Cerámicas arqueológicas. Perspectivas arqueométricas para su análisis e interpretación, B. Cremonte \& N. Ratto, Eds., pp. 169-185. San Salvador de Jujuy: Universidad Nacional de Jujuy.

López, M. A. \& D. Fiore, 2010. Técnicas relevantes para la caracterización de pigmentos arqueológicos: sus alcances analíticos y sus aplicaciones en casos de Argentina. En Cronograma del XVII Congreso Nacional de Arqueología Argentina, pp. 11-12. Mendoza: Universidad Nacional de Cuyo.

MARI, E., 1998. Los materiales cerámicos. Un enfoque unificador sobre las cerámicas tradicionales y avanzadas, los vidrios, los cementos, los refractarios y otros materiales inorgánicos no metálicos. Buenos Aires: Librería y Editorial Alsina.

Nielsen, A. E., 1997. Tiempo y cultura material en la Quebrada de Humabuaca. 700-1650 DC. San Salvador de Jujuy: Instituto Interdisciplinario de Tilcara.

— 2001. Evolución social en Quebrada de Humahuaca (AD 7001536). En Historia Argentina Prebispánica Vol.1, E. Berberián \& A. Nielsen, Eds., pp. 171-264. Córdoba: Editorial Brujas.

2010 [2007]. Celebrando con los antepasados. Arqueología del espacio público en Los Amarillos (Quebrada de Humabuaca, Jujuy, Argentina). Buenos Aires: Mallku Ediciones.

Palamarczuk, V., 2011. Un estilo y su época. El caso de la cerámica Famabalasto Negro Grabado del Noroeste Argentino. BAR International Series 2243. Oxford: Archaeopress.

PALMA, J. R., 1998. Curacas y señores. Una visión de la sociedad política prebispánica en la Quebrada de Humabuaca. Buenos Aires: Instituto Interdisciplinario de Tilcara, Universidad de Buenos Aires.

Rivolta, M. C., 1997. Revisión crítica de la obra de Bennett y colaboradores sobre la definición y asignación cronológica de algunos estilos cerámicos de la Quebrada de Humahuaca. Avances en Arqueología 3: 131-146, Buenos Aires.

Schweizer, F. \& A. Rinuy, 1982. Manganese Black as an Etruscan Pigment. Studies in Conservation 27: 118-123.

SHePARD, A. O., 1985. Ceramics for the Archaeologist. Washington D. C.: Carnegie Institution of Washington.

Striova, J.; C. Lofrumento, A. Zoppi \& E. M. Castellucci, 2006. Prehistoric Anasazi ceramics studied by micro-Raman spectroscopy. Journal of Raman Spectroscopy 37 (10): 1139-1145.

Turner, J. C., 1964. Descripción Geológica de la hoja 2C. Santa Victoria (Provincias de Salta y Jujuy). Carta geológico-económica de la República Argentina. Boletín N ${ }^{\circ} 104$. Buenos Aires: Ministerio de Economía de la Nación. 
